\title{
Synthese der Kohlenwasserstoffe.
}

(Fortsetzung von Band CL. Heft 1. pag. 73.)

\section{Vierter Theil.}

Bildung von Kohlenwasserstoffen durch trockne Destillation essigsaurer, buttersaurer und ölsaurer Salze und des Zuckers.

\section{Essigsaure Salze.}

Gleiche Theile trocknes essigsaures Natron und Natronhydratkalk wurden der trocknen Destillation unterworfen. Berthelot verwandte gegen $10 \mathrm{Kilogrm}$. des Natronsalzes zu diesen Versuchen. Die über Wasser im Recipienten der pneumatischen Wanne aufgefangenen Gase bestanden aus beinahe reinem Sumpfgas $\mathrm{C}^{2} \mathrm{H}^{4}$. Im Brom verdichteten sich ölbildendes Gas, Propylengas, Butylengas und Amylen. Berthelot konnte durch fractionirte Destillation trennen:

1) eine bei $145^{\circ} \mathrm{C}$. flüchtige Flüssigkeit, Brompropylen $\mathrm{C}^{6} \mathrm{H}^{6} \mathrm{Br}^{2}$, das Hauptproduct;

2) eine bei $160^{\circ} \mathrm{C}$. flüchtige Flüssigkeit, Brombutylen $\mathrm{C}^{8} \mathrm{H}^{8} \mathrm{Br}^{2}$

3) eine zwischen 175 und $180^{\circ} \mathrm{C}$. flüchtige Flüssigkeit, in kleiner Menge, Bromamylen $\mathrm{C}^{10} \mathrm{H}^{10} \mathrm{Br}^{2}$;

4) einige Tropfen einer unterhalb $140^{\circ} \mathrm{C}$. siedenden Flüssigkeit, ein Gemenge von Brompropylen und Bromäthylen $\mathrm{C}^{4} \mathrm{H}^{4} \mathrm{Br}^{2}$;

5) noch bei $200^{\circ} \mathrm{C}$. nicht flüchtige Bromide complicirter Kohlenwasserstoffe.

Das ölbildende Gas $\mathrm{C}^{4} \mathrm{H}^{4}$ wurde auch direct in dem Gasgemenge von der trocknen Destillation des essigsauren Natrons durch Behandlung des Gases mit Jod abgeschieden. Eine halbverkohlte Substanz blieb nach Hinwegnahme des überschüssigen Jods durch Natronlauge ungelöst. Mit Kalilauge gekocht, entwickelte diese Masse ölbildendes Gas in reinem Zustande, völlig durch Brom absorbirbar und eudiometrisch analysirt auf 1 Volum des Gases 2 Vol. Kohlensäure und 4 Vol. absorbirtes Sauerstoffgas liefernd.

Die Trennung des $\mathrm{C}^{4} \mathrm{H}^{4} \mathrm{~J}^{2}$ von den entsprechenden Jodiden des $\mathrm{C}^{6} \mathrm{H}^{6}, \mathrm{C}^{8} \mathrm{H}^{8}$ u. s. w. ist wichtig; die letzteren Kohlenwasserstoffe scheinen sich langsamer mit Jod zu verbinden als der $\mathrm{C}^{4} \mathrm{H}^{4}$ und ihre Verbindungen scheinen bei der eintretenden Temperaturerhöhung wieder zerlegt zu werden. Das gewonnene ölbildende Gas zeigte das 
charakteristische Verhalten gegen concentrirte Schwefelsäure, indem es von derselben stufenweise und fortdauernd (d'une manière graduelle et continue) mit Hülfe einer öfteren Bewegung (nach etwa 3000 Schüttelschlägen) absorbirt wurde. Im Ganzen ist die Menge des bei der trocknen Destillation des essigsauren Natrons gebildeten ölbildenden Gases ausserordentlich geringe.

Das Propylenbibromid $\mathrm{C}^{6} \mathrm{H}^{6} \mathrm{Br}^{2}$ von der trocknen Destillation des essigsauren Natrons stammend zeigte bei der Elementaranalyse 17,2 Proc. C, 2,9 Proc. H und 79,4 Proc. Brom (Verlust 0,5 Proc.). Die Formel $\mathrm{C}^{6} \mathrm{H}^{6} \mathrm{Br}^{2}$ verlangt $\mathrm{C}=17,8-\mathrm{H}=3,0-\mathrm{Br}=79,2$ Proc. Bei $2750 \mathrm{C}$. durch $\mathrm{Cu}, \mathrm{HO}$ und KJ zerlegt, lieferte es ein Gasgemenge aus $33 \mathrm{Vol}$. $\mathrm{C}^{6} \mathrm{H}^{6}, 21 \mathrm{Vol} . \mathrm{C}^{6} \mathrm{H}^{8}, 41 \mathrm{Vol} . \mathrm{H}$ und 5 Vol. $\mathrm{N}$.

Darstelhng von Propylalkohol. 20 Liter Gas von der trocknen Destillation des essigsauren Natrons mit Natronhydratkalk, ohne weiters über Wasser aufgefangen, wurden zur Entfernung der brenzlichen Dämpfe mit Schwefelsäure geschüttelt, die mit ihrem gleichen Volumen Wasser verdünnt worden war; darauf Liter auf Liter einige Minuten lang mit conc. Schwefelsäure. Nach dieser Operation zeigte die Schwefelsäure den für den Propylalkohol so charakteristischen Cypressengeruch. Vorsichtig mit Wasser verdünnt, mit kohlensaurem Kalk gesättigt, eingedampft, lieferte die Flüssigkeit ein sehr hygroskopisches Kalksalz mit den Eigenschaften des propylätherschwefelsauren Kalks. Mit benzoësaurem Kali gemengt und im Oelbade bei $220-240^{\circ} \mathrm{C}$. erhitzt, lieferte derselbe benzoësauren Propyläther, der den Geruch, den Siedepunct und die sonstigen Eigensehaften dieser Verbindung besass, aus welcher der Propylalkohol darstellbar ist.

In der brenzlichen Flüssigkeit von der trocknen Destillation des essigsauren Natrons suchte Berthelot vergeblich nach Holzgeist, Methyläther oder einer Methyloxydverbindung. Bei dieser Untersuchung fand derselbe, dass bei Einwirkung der concentrirten Schwefelsäure auf Aceton sich eine gewisse Menge von Propylengas $\mathrm{C}^{6} \mathrm{H}^{6}$ bildet.

Das Butylenbibromid $\mathrm{C}^{8} \mathrm{H}^{3} \mathrm{Br}^{2}$ aus dem Gase der trocknen Destillation des essigsauren Natrons durch Einwirkung von Brom gewonnen und durch fractionirte Destillation von dem beigemengten $\mathrm{C}^{6} \mathrm{H}^{6} \mathrm{Br}^{2}$ u. s. w. getrennt, verflüchtigte sich bei etwa $16^{\circ} \mathrm{C}$. Bei $275^{\circ} \mathrm{C}$. durch 
$\mathrm{Cu}, \mathrm{HO}+\mathrm{KJ}$ zerlegt, lieferte es ein Gasgemenge, worin 44 Vol. $\mathrm{C}^{8} \mathrm{H}^{8}, 12 \mathrm{Vol}$. $\mathrm{C}^{8} \mathrm{H}^{10}, 38 \mathrm{Vol}$. Wasserstoffgas und 6 Vol. Stickgas sich befanden (letzteres aus der Luft der Apparate).

Das Amylenbibromid $\mathrm{C}^{10} \mathrm{H}^{10} \mathrm{Br}^{2}$ beträgt nur wenig. Siedet bei $175-180^{\circ} \mathrm{C}$. unter beginnender Zersetzung. Die Analyse ergab 70,2 Procent Brom. Die Formel $\mathrm{C}^{10} \mathrm{H}^{10} \mathrm{Br}^{2}$ fordert 69,6 Proc. Brom. Durch Erhitzung bei $275^{\circ} \mathrm{C}$. mit $\mathrm{Cu}, \mathrm{HO}$ und $\mathrm{KJ}$ im Verschlossenen lässt sich daraus das Amylen $\mathrm{C}^{10} \mathrm{H}^{10}$ abscheiden, als flüchtige Flüssigkeit von dem charakteristischen Geruch und den übrigen Eigenschaften desselben.

Die unter den Producten der trocknen Destillation des essigsauren Natrons nachgewiesenen Kohlenwasserstoffe $\mathrm{C}^{4} \mathrm{H}^{4}, \mathrm{C}^{6} \mathrm{H}^{6}, \mathrm{C}^{8} \mathrm{H}^{8}$ und $\mathrm{C}^{10} \mathrm{H}^{10}$ sind in denselben ursprünglich vorhanden und entstehen nicht etwa erst durch Einwirkung des Broms auf Aceton oder andere pyrogene Flüssigkeiten dieser Destillation. Nur eine Spur von Bromoform $\mathrm{C}^{2} \mathrm{HBr}^{3}$ erzeugte sich bei Einwirkung des Broms auf Aceton. Es lieferte bei $275^{\circ} \mathrm{C}$. mit $\mathrm{Cu}$, $\mathrm{HO}$ und KJ erhitzt nur Sumpfgas, aber weder $\mathrm{C}^{4} \mathrm{H}^{4}$ noch $\mathrm{C}^{6} \mathrm{H}^{6}$. Unter den günstigsten Bedingungen beträgt der Kohlenstoff in den Kohlenwasserstoffen, die bei der trocknen Destillation des essigsauren Natrons entwickelt werden, nur 1/20 des Kohlenstoffs im letzteren.

\section{Anhang.}

Zersetzung des Alkohols und der Essigsäure in der Rothgluth.

Nach einer von Berthelot im Jahre 1851 (Annal. de Chim. et de Phys. 3. Sér. Novbr. 1851. Tom. XXXIII. pag. 295 - 302) veröffentlichten Untersuchung liefert Alkohol von $40^{\circ} \mathrm{B}$., bei Zersetzung seines Dampfes durch lebhaft rothglühende Porcellanröhren, die mit Bimssteinstückchen erfüllt sind: Naphthalin, Benzin, Phensäure, Essigsäure, Aldehyd, ölbildendes Gas, Wasserstoffgas, Kohlenoxydgas (wohl auch Sumpfgas), Kohle, gelbliche, im Aether zu gelber, blauschillernder Flüssigkeit lösliche Substanz, eine gelbröthliche knoblauchartig riechende Substanz; einen Körper der mit Salpetersäure behandelt, Moschusgeruch entwickelte u.s. w. Selbst nachdem die Gase 14 Waschflaschen durchströmt hatten, zeigten sie noch das Ansehen von Rauch und setzten in der Ruhe feste Theilchen ab.

Essigsäurehydrat, als Dampf durch glühende Röhren 
strömend, lieferte Naphthalim, Benzin $\mathrm{C}^{12} \mathrm{H}^{6}$, Aceton, eine gelblich-weisse feste Substanz, mit Dichroismus im Aether löslich; ein nach Moschus riechendes Product; Gase von empyreumatischem Geruch und reich an Kohlensäure. Merkwürdiger Weise zeigten diese Gase, selbst nachdem sie eine abgekühlte Verdichtungsflasche, sieben Waschflaschen, darunter eine mit Kalilauge, passirt hatten, welche letztere zu Ende des Versuchs noch alkalisch reagirte, noch saure Reaction.

$\mathrm{Zu}$ diesen länger bekannten Bildungsweisen des Naphthalins kommt jetzt die oben beschriebene Entstehung desselben aus Schwefelkohlenstoff oder Chlorkohlenstoff.

Bromoform, als Dampf über rothglühendes Eisen getrieben, liefert eine kleine Menge von Benzin C12 H6.

Absoluter Alkohol in einer luftleeren Glasröhre eingeschlossen, die er nur zu 1/5 erfüllt, liefert nach längerem Erhitzen bei starker Rothgluth eine beträchtliche Menge von Gas, so wie eine kleine Menge von Phensäure, aber keine Kohle.

Auch unter den flüssigen Producten der trocknen Destillation des essigsauren Natrons lässt sich eine kleine Menge von Phensäure nachweisen.

Naphthalin $\mathrm{C}^{20} \mathrm{H}^{8}$, Benzin $\mathrm{C}^{12} \mathrm{H}^{6}$ und Phensäure $\mathrm{C}^{12} \mathrm{H}^{5} \mathrm{O}, \mathrm{HO}$ lassen sich sonach aus Alkobol und Essigsäure darstellen; da die beiden letzteren aus mineralischen Stoffen erzeugt werden können, so ist hiermit auch die völlige Synthese jener complexen Verbindungen gegeben.

II. Trockne Destillation buttersaurer Salze.

Aus einer Retorte von glasurtem Steinzeug wurden 500 bis $1000 \mathrm{Grm}$. buttersaurer Baryt der trocknen Destillation unterworfen. Zwei gut gekühlte Vorlagen nahmen die flüssigen Producte der Destillation auf; an diese schloss sich eine Flasche mit Schwefelsäure, die mit ihrem gleichen Volumen Wasser verdünnt war; dieser folgte ein eiförmiger, mit Brom unter Wasser versehener Absorptionsapparat; daran reihte sich eine Waschflasche mit Natronlauge und den Schluss bildete ein mit luftfreiem absolutem Alkohol gefüllter Ballon, welcher zur Aufnahme der dem Sumpfgas analogen Kohlenwasserstoffe diente. Dieser Ballon wurde erst später vorgelegt, sobald die atmosphärische Luft der Apparate durch die nachströmenden pyrogenen Gase ausgetrieben war. An diesen Ballon schloss sich der Gasrecipient der pneumatischen Wanne an.

Die vom.Brom zurückgehaltenen Kohlenwasserstoffe 
wurden für sich untersucht, ebenso die aus der Alkohollösung durch Erhitzung wieder abgeschiedenen Hydrocarbüre.

a) Kohlenwasserstoffe, löslich in absolutem Alkohol, nicht absorbirbar durch Brom. Man entwickelte dieselben entweder durch Erhitzung der alkoholischen Lösung oder durch Zumischen des 2- bis 3fachen Volums Wasser zu derselben. Das aufgefangene Gas reinigte man durch Schütteln mit ein wenig Brom, dann mit etwas Kali und untersuchte es eudiometrisch. Eine Analyse ergab $50 \mathrm{Vol}$. Sumpfgas, $49 \mathrm{Vol}$. Aethylwasserstoffgas $\mathrm{C}^{4} \mathrm{H}^{6}$ und 1 Vol. Stickgas (aus der atmosphärischen Luft der Apparate).

b) Kohlenwasserstoffe mit Brom verbunden. Sie betrugen weit mehr, als die ïbrigen pyrogenen Flüssigkeiten der trocknen Destillation des buttersauren Baryts zusammengenommen. Durch fractionirte Destillation getrennt, lieferten sie:

1) eine Spur eines mit Wasser gemengten Bromids, unterhalb $130^{0} \mathrm{C}$. flüchtig (Aethylenbromid $\mathrm{C}^{4} \mathrm{H}^{4} \mathrm{Br}^{2}$ enthaltend);

2) Aethylenbromid $\mathrm{C}^{4} \mathrm{H}^{4} \mathrm{Br}^{2}$, bei $130^{0} \mathrm{C}$. flüchtig;

3) Propylenbromid $\mathrm{C}^{6} \mathrm{H}^{6} \mathrm{Br}^{2}$, bei $145^{0} \mathrm{C}$. flüchtig. Das bei weitem reichlichste Product der Destillation.

4) Butylenbromid $\mathrm{C}^{8} \mathrm{H}^{8} \mathrm{Br}^{2}$, bei $160^{0} \mathrm{C}$. und etwas darüber flüchtig;

5) Amylenbromid $\mathrm{C}^{10} \mathrm{H}^{10} \mathrm{Br}^{2}$ zwischen 175 und $180^{0}$ C. Hüchtig;

6) ein nicht flüchtiges Gemenge von Bromiden, etwa $1 / 5$ bis $1 / 4$ des Gesammtgewichts der vereinigten Bromkohlenwasserstoffe ausmachend; es scheint Hydrocarbüre, dichter als Amylen zu enthalten.

Die verschiedenen Bromide wurden durch Erhitzung bei $275^{\prime \prime} \mathrm{C}$. mit Kupfer, Wasser und Jodkalium zerlegt und so ölbildendes Gas, Propylengas, Butylengas, Butylwasserstoffgas erhalten. Das Brombutylen $\mathrm{C}^{8} \mathrm{H}^{8} \mathrm{Br}^{2}$, mit alkoholischer Kalihydratlösung destillirt, lieferte Monobrombutylen $\mathrm{C}^{8} \mathrm{H}^{7} \mathrm{Br}$, als neutrale Flüssigkeit, unlöslich im Wasser, etwas unter $100^{\circ} \mathrm{C}$. fliichtig. Das Bromamylen $\mathrm{C}^{10} \mathrm{H}^{10} \mathrm{Br}^{2}$, mit alkoholischer Kalilösung destillirt, lieferte das bei $120^{\circ} \mathrm{C}$. flüchtige Monobromamylen $\mathrm{C}^{10} \mathrm{H}^{9} \mathrm{Br}$.

Die Bromkohlenwasserstoffe, minder flüchtig als Bromamylen, wurden, analog dem Brombutylen und Bromamylen, durch Einwirkung alkoholischer Kalilösung in die èntsprechenden Monobromhydrocarbüre verwandelt, welche im Allgemeinen um 60 bis $80^{\circ} \mathrm{C}$. niedrigere 
Siedepuncte haben als die Bibromide. So war es möglich noch eine gewisse Menge von Monobromamylen $\mathrm{C}^{10} \mathrm{H}^{9} \mathrm{Br}$ $\mathrm{zu}$ gewinnen, aber auch noch Monobromhydrocarbüre mit höheren Siedepuncten und wahrscheinlich höherem Kohlenstoffgehalt. Die geringe Menge dieser Stoffe verhinderte ihre genauere Untersuchung.

Buttersäuredämpfe werden durch rothglühendes Eisen zerlegt, unter Bildung von Propylengas $\mathrm{C}^{6} \mathrm{H}^{6}$ und ölbildendem Gas.

Tribrombutyl $\mathrm{C}^{8} \mathrm{H}^{7} \mathrm{Br}^{3}$, (erhalten durch Erhitzung eines Gemenges von $1 \mathrm{Th}$. Buttersäure mit $15 \mathrm{Th}$. $\mathrm{PBr}^{5}$ bei $100^{\circ} \mathrm{C}$., 200 Stunden lang in zugeschmolzenen Gefässen, Destillation des Products und Aufsammeln des zwischen 185 und $190^{\circ} \mathrm{C}$. Uebergehenden) lässt sich in Butylwasserstoff $\mathrm{C}^{8} \mathrm{H}^{10}$ verwandeln, durch Erhitzung mit Kupfer und wasserfreiem Fünffach-Schwefelwasserstoff in lufttreien zugeschmolzenen Glasröhren bei $275^{\circ} \mathrm{C}$. Doch ist derselbe mit viel Wasserstoffgas gemengt.

\section{Destillation des ölsauren Kalks.}

Sie liefert reichliche Mengen Propylen, Butylen und Amylen. Man mengt 1 Kilogrm. Oelsäure des Handels mit 300 Grm. gelöschtem Kalk und das so gebildete Kalksalz mit $300 \mathrm{Grm}$. Natronhydratkalk und bringt das Gemenge in eine steinzeugne Retorte von 2 Liter Capacität. An diese fügt man 1) eine Reihe von 2 bis 3 sorgfailtig gekühlten leeren Flaschen; 2) eine Literflasche, enthaltend Schwefelsäure, mit ihrem gleichen Volum Wasser verdünnt; 3) zwei eiförmige Absorptionsflaschen, deren erste $750 \mathrm{Grm}$. Brom und $100 \mathrm{Grm}$. Wasser, deren zweite nur 250 Grm. Brom und ebenfalls 100 Grm. Wasser enthält. Die erste Bromflasche wird gut abgekühlt; 4) eine Literflasche, mit müssig verdünnter Natronlauge.

Man erhitzt die Retorte über freiem Feuer vorsichtig. Bald beginnen die Flüssigkeiten und die Gase sich zu entwickeln und zwei Stunden darauf ist die Operation beendet. Man stellt die in den ersten Condensatoren angesammelten Flüssigkeiten bei Seite und nimmt nur die beiden Bromflissigkeiten in Arbeit. Das Brom der ersten Flasche ist völlig verschwunden und in farblose Bromkohlenwasserstoffe verwandelt. Man mischt beide Bromflüssigkeiten mit einander, fügt verdünnte Natronlauge hinzu, um das überschüssige Brom und die gebildete Bromwasserstoffsäure aufzunehmen, so wie um einige Bromverbindungen zu zerstören, welche die Kohlenwas- 
serstoffbromide $\mathrm{C}^{2 \mathrm{n}} \mathrm{H}^{2 \mathrm{n}} \mathrm{Br}^{2}$ verunreinigen, schüttelt dann mit Wasser und stellt die öligen Bromide bei Seite. Man wiederholt zweimal diese Destillation des ölsauren Kalks mit Natronkalk und erhält schliesslich aus 3 Kilogrm. Oelsäure und 3 Kilogrm. Brom nahezu 2 Kilogrm. Flüssigkeiten in den ersten Condensationsgefässen und mehr als $1200 \mathrm{Grm}$. Bromkohlenwasserstoffe. Nach einer Reihe systematischer fractionirter Destillationen liefern diese letzteren annähernd:

600 Grm. Brompropylen $=\mathrm{C}^{6} \mathrm{H}^{6} \mathrm{Br}^{2}$,

100 Bromäthylen $=\mathrm{C}^{4} \mathrm{H}^{4} \mathrm{Br}^{2}$,

$100 "$ Brombutylen $=\mathrm{C}^{8} \mathrm{H}^{8} \mathrm{Br}^{2}$,

$50 \pi$ Bromamylen $=\mathrm{C}^{10} \mathrm{H}^{10} \mathrm{Br}^{2}$ und

200 bis $300 \mathrm{Grm}$. Bromkohlenwasserstoffe von höherem Atomgewicht als Bromamylen und nicht unzersetzt flüchtig.

Die systematischen fractionirten Destillationen führt man aus, wie folgt:

Das ursprüngliche Gemenge der Bromkohlenwasserstoffe bringt man in eine tubulirte Retorte und destillirt mit eingesenktem Thermometer. Man sammelt für sich:

1) die Producte, welche unterhalb $135^{\circ} \mathrm{C}$. flüchtig sind;

2) die zwischen $135^{\circ}$ und $155^{\circ} \mathrm{C}$. flüchtigen Producte;

3) die zwischen $155^{\circ}$ und $170^{\circ} \mathrm{C}$. flüchtigen Oele;

4) die zwischen $170^{\circ}$ und $190^{\circ} \mathrm{C}$. flüchtigen Producte.

Die ersteren bestehen hauptsächlich aus Wasser und Bromäthylen; die zweiten aus Brompropylen; die dritten aus Brombutylen und die vierten aus Bromamylen; aber alle sind sehr weit davon entfernt, rein zu sein. Jedes derselben enthält neben dem Hauptbromid auch noch alle übrigen, namentlich ist allen Propylenbromid in beträchtlicher Menge beigesellt.

Man rectificirt zuerst das vierte Product, welches diesmal schon bei 140 bis $1500 \mathrm{C}$. zu sieden beginnt. Alles, was bis auf $150^{\circ} \mathrm{C}$. überdestillirt, giesst man zum zweiten Product, das von $155^{\circ} \mathrm{C}$. bis $170 \mathrm{C}$. Destillirende giebt man zum dritten Product und ohne weiter zu destilliren bringt man den Retortenrückstand in Reserve als unreines Bromamylen.

Nun rectificirt man das dritte Product, welches jetzt schon bei $135^{\circ} \mathrm{C}$. zu sieden beginnt. Man vereinigt mit dem ersten Producte Alles, was zwischen 135 und $140^{\circ} \mathrm{C}$. übergeht; mit dem zweiten Product das zwischen 140 und $155^{\circ} \mathrm{C}$. Destillirende und stellt das zwischen 155 und $170^{\circ} \mathrm{C}$. Uebergehende als unreines Brombtuylen bei 
Seite. Der Retortenrückstand ist unreines Bromamylen und wird zu dem schon in Reserve gestellten gegeben.

Jetzt rectificirt man das erste Product. Anfangs geht ein wenig Wasser und etwas Aethylenbromid über, bis zu 1350C.; man stellt es bei Seite. Dंas zwischen 135 und $155^{\circ} \mathrm{C}$. Uebergehende mischt man zum zweiten Product; das zwischen 155 und $170^{\circ} \mathrm{C}$. Destillirende zum unreinen Brombutylen und den Retortenrückstand zum unreinen Bromamylen.

Man beendigt die Reihe dieser Destillationen mit der-Rectification des zweiten Products, dem reichlichsten von allen. Man destillirt bis zum Siedepunct $140^{\circ} \mathrm{C}$. und giesst das Destillat zum unreinen Bromäthylen; das zwischen 140 bis $165^{\circ} \mathrm{C}$. Uebergehende stellt man als unreines Brompropylen bei Seite; das zwischen 155 und $170^{\circ} \mathrm{C}$. Destillirende giebt man zum unreinen Brombutylen und den Retortenrïickstand giesst man zum unreinen Bromamylen.

So hat man, ohne genöthigt gewesen zu sein, irgend etwas wegzuschütten, die vier Bromkohlenwasserstoffe: Aethylen-, Propylen-, Butylen- und Amylenbibromid annähernd von einander geschieden.

Es folgt nun die Reinigung der einzelnen Oele: Man rectificirt das unreine Propylenbromid und sammelt nur das zwischen 145 und $150^{\circ} \mathrm{C}$. Uebergehende; es ist beinahe reines $\mathrm{C}^{6} \mathrm{H}^{6} \mathrm{Br}^{2}$. (Auf dieses Product bezieht sich die obige Gewichtsangabe.) Eine neue Destillation bei $148^{\circ} \mathrm{C}$. liefert das Propylenbibromid völlig rein.

Nun rectificirt man das unreine Brombutylen und sammelt nur das zwischen 160 bis $165^{\circ} \mathrm{C}$. Destillirende.

Man rectificirt das unreine Bromamylen und sammelt nur das bei $180^{\circ} \mathrm{C}$. Uebergehende.

Das unreine Bromäthylen reinigt man durch Destillation und Sammeln des bei $130^{\circ} \mathrm{C}$. Uebergehenden. Es ist die Gewinnung dieses $\mathrm{C}^{4} \mathrm{H}^{4} \mathrm{Br}^{2}$ nicht vortheilhaft. Aber $\mathrm{C}^{6} \mathrm{H}^{6} \mathrm{Br}^{2}, \mathrm{C}^{8} \mathrm{H}^{8} \mathrm{Br}^{2}$ und $\mathrm{C}^{10} \mathrm{H}^{10} \mathrm{Br}^{2}$ lassen sich aus der Oelsäure vortheilhafter gewinnen, als aus den festen fetten Säuren oder aus Amylalkohol.

\section{Destillation des Zuckers.}

1 Kilogrm. gemeiner Zucker oder Krümelzucker, mit seinem gleichen Gewicht Natronhydratkalk gemengt der trocknen Destillation unterworfen, lieferte in dem zuletzt beschriebenen ähnlichen Apparaten einige Grammen ölige Bromkohlenstoffe in den Bromvorlagen. Durch 
mehrere Destillationen sammelte $\mathrm{B}$ erthelot eine gewisse Menge derselben und isolirte daraus Bromäthylen $\mathrm{C}^{4} \mathrm{H}^{4} \mathrm{Br}^{2}$, Brompropylen $\mathrm{C}^{6} \mathrm{H}^{6} \mathrm{Br}^{2}$ und Brombutylen $\mathrm{C}^{8} \mathrm{H}^{8} \mathrm{Br}^{2}$. Aus jedem dieser Bromide regenerirte er durch umgekehrte Substitution die entsprechenden Kohlenwasserstoffe und bewies durch eudiometrische Analyse deren Zusammensetzung. Unter den pyrogenen Flüssigkeiten dieser Destillation suchte er vergeblich noch Allylalkohol und Allyläther. Da das Aethylengas $\mathrm{C}^{4} \mathrm{H}^{4}$ leicht in Alkohol übergeführt werden kann, so hat man die Bildung von Alkohol aus Zucker ohne Gährung hiermit in seiner Hand.

\section{Schlussbemerkungen.}

Bis jetzt stellte man die Kohlenwasserstoffe immer nur durch Zerstörung organischer Verbindungen dar. Bei dieser gewöhnlich durch Wärme bewirkten Zersetzung theilen sich die Elemente der Verbindungen in zwei ungleiche Portionen; der eine Theil des Kohlenstoffs und Wasserstoffs wird durch den Sauerstoff der Substanz völlig verbrannt, während der andere Theil in Form von verbrennlicheren Verbindungen auftritt, als der zersetzte Körper selbst war. Diese Producte der Zersetzung sind im Allgemeinen einfacher in der Zusammensetzung, einfacher hinsichtlich der Zahl der Kohlenstoff-Aequivalente, als diejenigen, woraus sie entstanden. Allein dieses Verfahren ist rein analytisch; es erlaubt nicht, den ersten Schritt der Synthese zu thun; nämlich die Bildung der Kohlenwasserstoffe aus ihren Elementen, da es die Existenz von Kohlenwasserstoff - Verbindungen voraussetzt. Aber diese Bildung aus den Elementen soll ja eben erzielt werden.

Das Sumpfgas $\mathrm{C}^{2} \mathrm{H}^{4}$ wurde zuerst als freiwilliges Zersetzungsproduct organischer Ueberreste gesammelt; später lernte man dasselbe durch die trockne Destillation organischer Substanzen, namentlich der essigsauren Salze bereiten.

Das ölbildende Gas $\mathrm{C}^{4} \mathrm{H}_{4}$, das Product der trocknen Destillation einer grossen Anzahl organischer Substanzen, bereitete man meistens aus Alkohol und diesen aus Zucker.

Das Propylengas $\mathrm{C}^{6} \mathrm{H}^{6}$, das Butylen $\mathrm{C}^{8} \mathrm{H}^{8}$, das Amylen und analoge , Kohlenwasserstoffe bereitete man mit Hülfe der entsprechenden Alkohole, durch trockne Destillation der Salze organischer Säuren, welche sämmtlich eine complicirtere Zusammensetzung haben, als die daraus dargestellten Kohlenwasserstoffe. 
Das Naphthalin $\mathrm{C}^{20} \mathrm{H}^{8}$ und das Benzin $\mathrm{C}^{2} \mathrm{H}^{6}$ bereitete man ebenfalls aus organischen Verbindungen.

Immer aber waren die letzteren complexere Verbindungen, als die daraus erzeugten Kohlenwasserstoffe.

Berthelot's Methode diese Verbindungen darzustellen, ist eine der bisher üblichen gerade entgegengesetzte. Sie gestattet, die Hydrocarbüre und die daraus hervorgehenden Alkohole aus den Elementen Kohlenstoff und Wasserstoff und Sauerstoff zusammenzusetzen.

Die mitgetheilte Arbeit liefert den Beweis für diese Behauptung.

Das Sumpfgas $\mathrm{C}^{2} \mathrm{H}^{4}$ wird durch Destillation des ameisensauren Baryts gewonnen, welcher selbst aus Kohlenoxyd entstand, das seine Bildung der Einwirkung des Eisens auf kohlensauren Baryt verdankte. Auch aus dem Schwefelkohlenstoff $\mathrm{C}^{2} \mathrm{~S}^{4}$ gewinnt Berthelot das Sumpfgas.

Das ölbildende Gas $\mathrm{C}^{4} \mathrm{H}^{4}$ entsteht ebenfalls aus dem durch unorganische Verbindungen erzeugten ameisensauren Baryt durch die trockne Destillation; ferner durch Einwirkung des Wasserstoffgases und Eisens oder Kupfers auf Schwefelkohlenstoff bei Rothglühhitze.

Das Propylengas $\mathrm{C}^{6} \mathrm{H}^{6}$ bildet sich bei der trocknen Destillation desselben ameisensauren Baryts.

Das Butylen $\mathrm{C}^{8} \mathrm{H}^{8}$ und Amylen $\mathrm{C}^{10} \mathrm{H}^{10}$ werden durch trockne Destillation des essigsauren Natrons erzeugt, dessen Essigsäure ein Oxydationsproduct des Alkohols ist, den man mit jenem, aus unorganischen Stoffen gewonnenen Aethylengas $\mathrm{C}^{4} \mathrm{H}^{4}$ componiren kann.

Das Naphthalin $\mathrm{C}^{20} \mathrm{H}^{8}$ wird aus Schwefelkohlenstoff dargestellt, aus Alkohol, aus Essigssäure; das Benzin $\mathrm{C}^{12} \mathrm{H}^{6}$ ebenfalls aus Alkohol und Essigsäure.

Der Ausgangspunct der Synthese der organisehen Verbindungen ist hiermit gesichert. Es bleibt nun noch übrig, von den Kohlenwasserstoffen zu den Sauerstoffverbindungen derselben aufzusteigen. Berthelot hat diesen Weg mit Glück eingeschlagen und die Hydrocarbüre in die ihnen entsprechenden Alkohole umgewandelt.

Wie geschah seither die Darstellung der Alkohole? Der Methylalkohol oder Holzgeist $\mathrm{C}^{2} \mathrm{H}^{4} \mathrm{O}^{2}$ wurde aus den Producten der trocknen Destillation des Holzes abgeschieden.

Den Weingeist $\mathrm{C}^{4} \mathrm{H}_{6} \mathrm{O}^{2}$ gewann man aus dem Gährungsproduct des Zuckers.

Der Propylalkohol $\mathrm{C}^{6} \mathrm{H}^{8} \mathrm{O}^{2}$, Butylalkohol $\mathrm{C}^{8} \mathrm{H}^{10} \mathrm{O}^{2}$ 
und Amylalkohol $\mathrm{C}^{10} \mathrm{H}^{12} \mathrm{O}^{2}$ aus den Nebenproducten der geistigen Gährung des Zuckers;

Caprylalkohol $\mathrm{C}^{16} \mathrm{H}^{18} \mathrm{O}^{2}$ durch Destillation des Ricinusöls mit Alkalien;

Aethylalkohol $\mathrm{C}^{32} \mathrm{H}^{34} \mathrm{O}^{2}$ aus Wallrath; Capryl- und Melissylalkohol aus Wachs.

Berthelot lehrte nun, statt dieser rein analytischen Methoden, synthetische Methoden der Alkoholgewinnung. So bildete er Methylalkohol $\mathrm{C}^{2} \mathrm{H}^{4} \mathrm{O}^{2}$ aus Sumpfgas $\mathrm{C}^{2} \mathrm{H}^{4}$, indem er darin zuerst 1 Aeq. H durch 1 Aeq. Chlor ersetzte, dann das gebildete Chlormethyl $\mathrm{C}^{2} \mathrm{H}^{3} \mathrm{Cl}$ durch Kalihydrat zerlegte.

Weingeist $\mathrm{C}^{4} \mathrm{H}^{6} \mathrm{O}^{2}$ und Propylalkohol $\mathrm{C}^{6} \mathrm{H}^{8} \mathrm{O}^{2}$ durch Verbindung des $\mathrm{C}^{4} \mathrm{H}^{4}$ und $\mathrm{C}^{6} \mathrm{H}^{6}$ mit concentrirter Schwefelsäure und Zerlegung der erzeugten Aetherschwefelsäuren durch Wasser. Die Elemente des letzteren bleiben nun mit den Kohlenwasserstoffen verbunden und liefern die beiden Alkohole.

Propylalkohol $\mathrm{C}^{6} \mathrm{H}^{8} \mathrm{O}^{2}$, Amylalkohol $\mathrm{C}^{10} \mathrm{H}^{12} \mathrm{O}^{2}$, Caprylalkohol $\mathrm{C}^{16} \mathrm{H}^{18} \mathrm{O}^{2}$, Aethylalkohol $\mathrm{C}^{32} \mathrm{H}^{34} \mathrm{O}^{2}$ und wahrscheinlich alle übrigen können nach Berthelot vermittelst ihrer Haloidäther erhalten werden, welche ihrerseits aus der Vereinigung der Wasserstoffsäuren mit den entsprechenden Kohlenwasserstoffen sich gewinnen lassen. Z.B. $\mathrm{C}^{6} \mathrm{H}^{6}+\mathrm{HCl}=\mathrm{C}^{6} \mathrm{H}^{7} \mathrm{Cl} ; \mathrm{C}^{8} \mathrm{H}^{8}+\mathrm{HBr}=\mathrm{C}{ }^{8} \mathrm{H}^{9} \mathrm{Br}$; $\mathrm{C}^{10} \mathrm{H}^{10}+\mathrm{HJ}=\mathrm{C}^{10} \mathrm{H}^{11} \mathrm{~J}$. Und $\mathrm{C}^{6} \mathrm{H}^{7} \mathrm{Cl}+\mathrm{KO}, \mathrm{HO}=$ $\mathrm{C}^{6} \mathrm{H}^{8} \mathrm{O}^{2}+\mathrm{KCl} ; \mathrm{C}^{8} \mathrm{H}^{9} \mathrm{Br}+\mathrm{KO}, \mathrm{HO}=\mathrm{C}^{8} \mathrm{H}^{10 \mathrm{O}^{2}}+\mathrm{KBr}$; $\mathrm{C}^{10} \mathrm{H}^{11 \mathrm{~J}}+\mathrm{KO}, \mathrm{HO}=\mathrm{C}^{10} \mathrm{H}^{12 \mathrm{O}^{2}}+\mathrm{KJ}$ u. s. w.

Man kann also nach Berthelot die totale Synthese aller dieser Alkohole ausführen, deren Kohlenwasserstoffe aus den Elementen $\mathrm{C}$ und $\mathrm{H}$ dargestellt wurden, nämlich die totale Synthese von Methyl-, Aethyl-, Propyl-, Butyl-, Amylalkohol u. s.w. Aber diese Alkohole sind durch die Arbeiten der Chemiker unserer Zeit der Ausgangspunct der meisten übrigen organischen Verbindungen geworden.

Die Ausführung der totalen Synthese der Kohlenwasserstoffe und der Alkohole heisst sonach nichts anderes, als die völlige Zusammensetzung einer beinahe unendlichen Zahl organischer Verbindungen, sowohl natürlicher als künstlicher, mit Hülfe der einfachen Stoffe, ihrer Elemente. (M. Berthelot; Ann. de Chim. et de Phys. 3. Sér. Mai et Juni 1858. T. LIII. p. 69-208.)

Dr. H. Ludwig. 